$1.8 \%$ over the entire Jomon period. Violent injuries were evenly distributed across the country, and the researchers found no hotspots of violence that might indicate warfare.

The findings are inconsistent with the idea that warfare is inherent in human nature, the authors say.

Biol. Lett. 12, 20160028 (2016) MICROBIOLOG

\section{Salmonella live on thanks to toxin}

A toxin protein secreted by typhoid-causing bacteria seems to keep infected hosts alive, allowing the bacteria to persist in the body.

Salmonella enterica Typhi

(S. Typhi), which causes typhoid fever in humans but not in mice, produces a DNA-damaging protein. To study this toxin's role in mouse infections, Teresa Frisan at the Karolinska Institute in Stockholm and her team engineered another strain of S. enterica, called $S$. Typhimurium (which causes illness in mice but does not normally make the typhoid toxin) to make the part of the toxin that damages host DNA.

Mice infected with the toxin-producing strain were less likely to become severely ill and had less gut inflammation than did mice infected with a control strain. Toxin-producing bacteria could still be found in the livers of mice six months after infection, in contrast to the control strain, which was undetectable in mice that survived the initial infection.

PLoS Pathog. 12, e1005528

(2016)

\section{Sugars made in} simulated space

A key sugar found in DNA has been created in the laboratory under conditions similar to those around comets.

Ribose forms the backbone of DNA and RNA, but its ancient origin remains a mystery. Cornelia Meinert and Uwe Meierhenrich of the University of Nice Sophia Antipolis in France and their team created an artificial comet by condensing water, methanol and ammonia in a vacuum chamber at $-195^{\circ} \mathrm{C}$. The material was irradiated with ultraviolet light to simulate the formation of cometary ices. The residues that formed when the material was warmed to room temperature contained ribose and other, similar sugars in amounts that were much greater than just trace levels.

The authors suggest that comets and meteorites are the source of organic molecules that made life possible on Earth.

Science 352, 208-212 (2016)

\section{Molecule melts away cholesterol}

The next weapon against heart disease could be a compound that is currently used to make drugs more soluble.

In atherosclerosis, plaques containing crystallized cholesterol clog up blood vessels. Eicke Latz of the University Hospital in Bonn, Germany, and his colleagues tested a compound called 2-hydroxypropyl$\beta$-cyclodextrin, which increases the solubility of cholesterol, to see whether it reduced the plaques. They found that plaques shrank in atherosclerotic mice that had consumed cyclodextrin (blood vessel pictured left, cholesterol

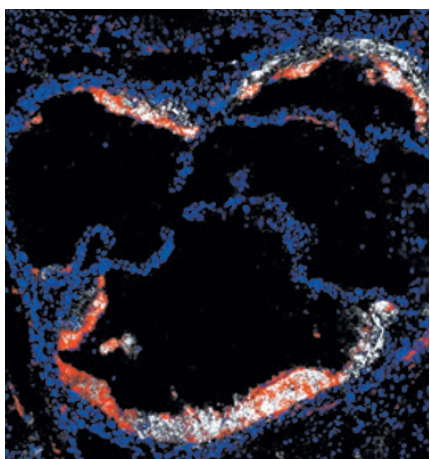

crystals in white), compared with plaques in the blood vessels of untreated animals (pictured right).

The drug bound to and dissolved the cholesterol crystals. It also increased cholesterol metabolism in immune cells called macrophages, which usually contribute to atherosclerosis by triggering inflammation in response to excess cholesterol. Cyclodextrin reprogrammed the cells in plaques, leading to increased transport of the dissolved cholesterol away from the plaques, and reducing harmful inflammation. Some of the same effects were seen in human plaque samples treated with the compound.

Sci. Transl. Med. 8, 333ra50 (2016)

\section{BIOCHEMISTR}

\section{Bioplastic made from glucose}

Researchers have combined three biochemical pathways to produce a biodegradable plastic from glucose in the laboratory.

Some industrial chemicals are made by microorganisms in bioreactors, but reengineering the organisms metabolic pathways to boost yields is challenging, so researchers are keen to find cell-free production methods. Polyhydroxybutyrate bioplastic (PHB) can be made without cells, but the process requires expensive starting materials. To lower costs, James Bowie and his colleagues at the University of California, Los Angeles, devised a cell-free way to make PHB out of glucose.

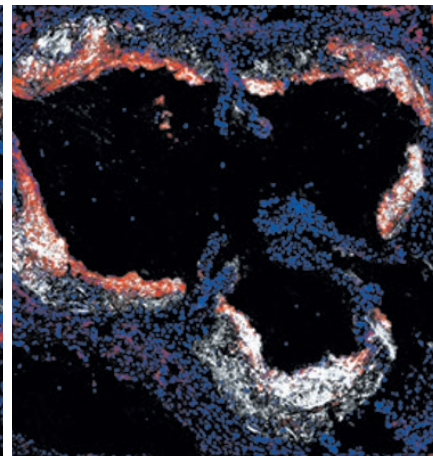

They designed a synthetic biochemical cycle comprising parts of three enzyme-driven pathways. Using two different concentrations of glucose, the team generated $\mathrm{PHB}$ at $86 \%$ and $94 \%$ yields. The yields and production rates were close to those required by industry.

With further improvements, this synthetic biochemistry approach could be used to produce chemicals at low cost, the authors say.

Nature Chem. Biol. http://dx.doi. org/10.1038/nchembio.2062 (2016)

NEUROIMMUNOLOG

\section{Protein linked to immune privilege}

A protein found in neurons helps to limit inflammation in the central nervous system (CNS), contributing to the system's specialized immune environment.

The CNS can stave off excessive inflammation. This 'immune privilege' has been attributed to the blood-brain barrier that restricts the entry of certain immune cells, but recent work has suggested a role for other cells and molecules. Lieping Chen at Yale University in New Haven, Connecticut, and his colleagues found that SALM5, a protein involved in neuronal growth and development, inhibits inflammation in the mouse CNS. In animals with an autoimmune CNS disease, blocking a receptor for SALM5 or treating with an antibody against SALM5 aggravated symptoms. Applying SALM5 to certain immune cells in a lab dish suppressed their response to a pro-inflammatory molecule.

The findings could lead to treatments for inflammatory neurological diseases, the authors suggest.

Sci. Adv. 2, e1500637 (2016)

\section{- NATURE.COM}

For the latest research published by Naturevisit: 